\title{
Momordica and Pycnogenol Can Tolerate Imazamox Induced Toxicity in L929 Cells Line: In Vitro Study ${ }^{\dagger}$
}

\author{
Ali Taghizadehghalehjoughi ${ }^{1}$ and Betul Cicek ${ }^{2, *}$ \\ 1 Department of Pharmacology and Toxicology, Ataturk University Faculty of Veterinary Medicine, \\ 25100 Erzurum, Turkey; ali.tgzd@atauni.edu.tr \\ 2 Department of Physiology, Ataturk University Faculty of Medicine, 25100 Erzurum, Turkey \\ * Correspondence: bcicek_24@hotmail.com; Tel.: 0544-387-6705 \\ + Presented at the 2nd International Cell Death Research Congress, Izmir, Turkey, 1-4 November 2018. \\ Published: 11 December 2018
}

\begin{abstract}
Undesirable side effects that result from the random use of herbicides in developing countries are widespread. Imazamox is a widely used herbicide and has toxic effect on humans. Momordica charantia has been reported to possess many benefits and medicinal properties. Pycnogenol (PYC) is a natural plant extract from the bark of Pinus pinaster Aiton and has potent antioxidant activities. The aim of this study was to evaluate the protective effects of pycnogenol and momardica on induced imazamox toxicity effects on L929 fibroblast cell line. L929 fibroblast cells were cultured in the appropriate culture medium. Toxic concentration of imazamox $250 \mu \mathrm{M}$, were administered $30 \mathrm{~min}$ prior to momordica and pycnogenol $\left(10^{-1}-10^{-5}\right.$ concentration) on L929 fibroblast cell line for $24 \mathrm{~h}$. The cell viability assay was determined by using MTT test. TAC-TOS analysis were used to evaluate antioxidant and oxidant status. According to our study pycnogenol high dose showned protective effect whereas momordica low dose showned protective effect $p<$ 0.05. In current study pycnogenol increased TAC capacity in high dose but in lower dose pycnogenol did not show any promise $(p<0.05)$. Momordica results showed correlation with MTT result. According to this analyse momordica only in low dose increased anti oxidant capacity and in addition, only in low dose TOS level were decreased $(p>0.05)$. In conclusion momordica and pycnogenol showed promise to reduced imazamox toxicity.
\end{abstract}

Keywords: L929; momordica; imazomox

\section{Introduction}

In today's world, the use of herbicide has become so important that their use is inextricably linked with improvement of human welfare. Despite of all the advantages of herbicides, there is worldwide concern about their use because of not specific targeting of herbicides. Imazamox is a member of the imidazolinone class of chemicals including imazapic, imazapyr, imazethapyr, imazamethabenz and imazaquinine, which are part of the Clearfield cropping system [1]. Phenolic compounds found in daily diets have various significant effects on diseases and experimental data are gathering regarding phenolic compounds as natural phytochemical antioxidants important for human health. Momordica charantia also called as bitter melon and kugua is widely cultivated in China, East Africa and South America. This plant has been traditionally used in folk medicine due to its an extensive range of nutritive and pharmacological properties against many diseases. Momordica has major chemical constituents, which are triterpenoids and their glycosides. A growing body of scientific literature has revealed that triterpenoids from Momordica are associated with their freeradical scavenging activity [2]. Pycnogenol (PYC), a family of flavonoids extracted from French maritime pine bark (Pinus pinaster Aiton, synonym Pinus maritima Mill.), which consisting of several 
types of phenolic compounds, condensed flavonoids and phenolic acids. It has been reported to have diverse beneficial pharmacological effects such as potent antioxidants, ROS scavenging and antiinflammatory properties [3]. In this study we aimed that investigation of protective effect of pycnogenol and momardica on induced imazamox toxicity on L929 fibroblast cell line.

\section{Material and Methods}

Cell Culture: L929 fibroblast cells were cultured in Dulbecco's modified Eagle's medium (DMEM) (Gibco Sigma, St. Louis, MO, USA) supplemented with $10 \%$ fetal bovine serum (FBS; Invitrogen, Carlsbad, CA, USA), $100 \mathrm{IU} / \mathrm{mL}$ penicillin and $100 \mu \mathrm{g} / \mathrm{mL}$ of streptomycin in $75 \mathrm{~cm} 2$ culture flasks. Cells were maintained in a humidified atmosphere with $5 \% \mathrm{CO}_{2}$ at $37^{\circ} \mathrm{C}$ with medium renewal 2-3 times a week depending on cell density. Determination of Cell Viability: The viability of fibroblast cell was examined by using 3-(4,5-dimethylthiazol-2-yl)-2,5-diphenyltetrazolium bromide (MTT) assay on the microplate reader (Gibco Sigma, St. Louis, MO, USA). Different concentration of imazamox $(250 \mu \mathrm{M})$, momordica and pycnogenol $\left(10^{-1}-10^{-5}\right.$ concentration) was applied on L929 fibroblast cells line for $24 \mathrm{~h} \mathrm{[4]}$. TAC and TOS: Spectrophotometric analysis was used for estimation of oxidative stress levels. Total oxidant status and TAC levels were measured after $24 \mathrm{~h}$ exposure to treatments. The cell mediums were collected when the experiments were completed. According to the manufacture procedure, TOS $\left(\mathrm{H}_{2} \mathrm{O}_{2}\right.$ Equiv/mmol L-1) absorbance were evaluated at $530 \mathrm{~nm}$ and TAC (Trolox Equiv/mmol L-1) at $660 \mathrm{~nm}$ was evaluated (Rel assay, Antep, Turkey) [5,6]. Statistically Analysis: The statistical analysis was done by Mann-Whitney U test by using the SPSS 22.0 software (IBM company, Turkey). $p<0.05$ was considered as statistically important distinction for whole test.

\section{Results}

MTT test: To determine cell viability, MTT assay was performed after $24 \mathrm{~h}$ exposure of imazamox, momordica and pycnogenol for fibroblast cell culture. (Figure 1).
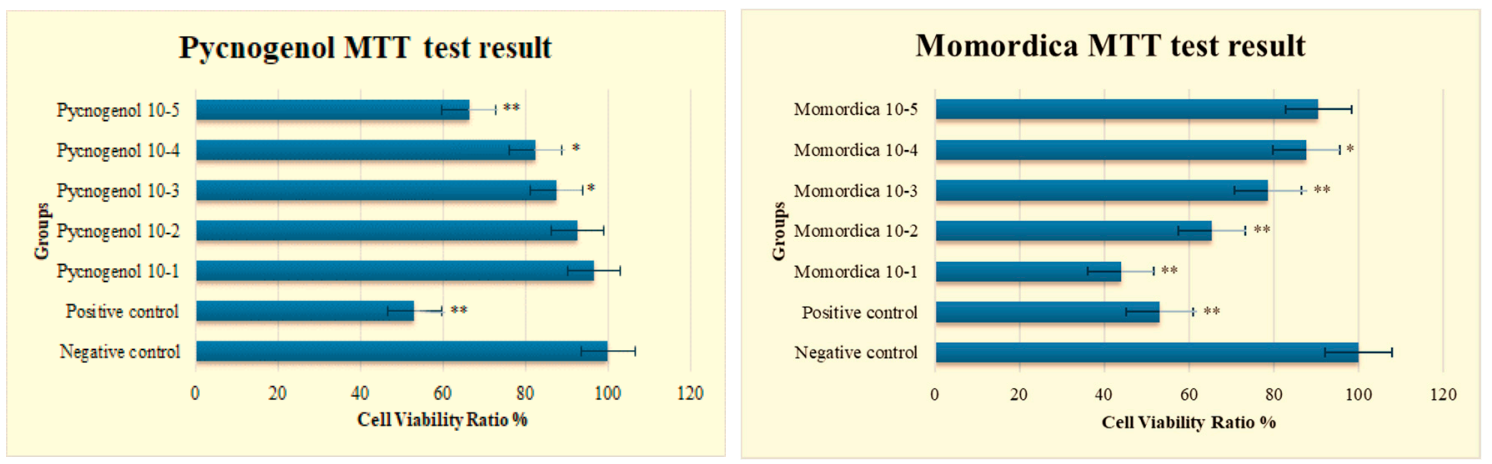

Figure 1. In vitro viability ratio of pycnogenol and momordica protective effect on induced imazamox toxicity effect on fibroblast cells $\left(n=6\right.$ /group) were shown. ${ }^{*}$ Significant differences at $p<0.05$ compared to control group; ${ }^{* *}$ Significant differences at the $p<0.001$ compared to control group.

According to our study pycnogenol high dose showned protective effect whereas momordica low dose showned protective effect $p<0.05$. TAC and TOS: In current study pycnogenol increased TAC capacity in high dose but in lower dose pycnogenol did not show any promise $(p<0.05)$. Momordica results showed correlation with MTT result. According to this analyses momordica only in low dose increased antioxidant capacity and in addition, only in low dose TOS level were decreased $(p>0.05)$. (Figure 2). 

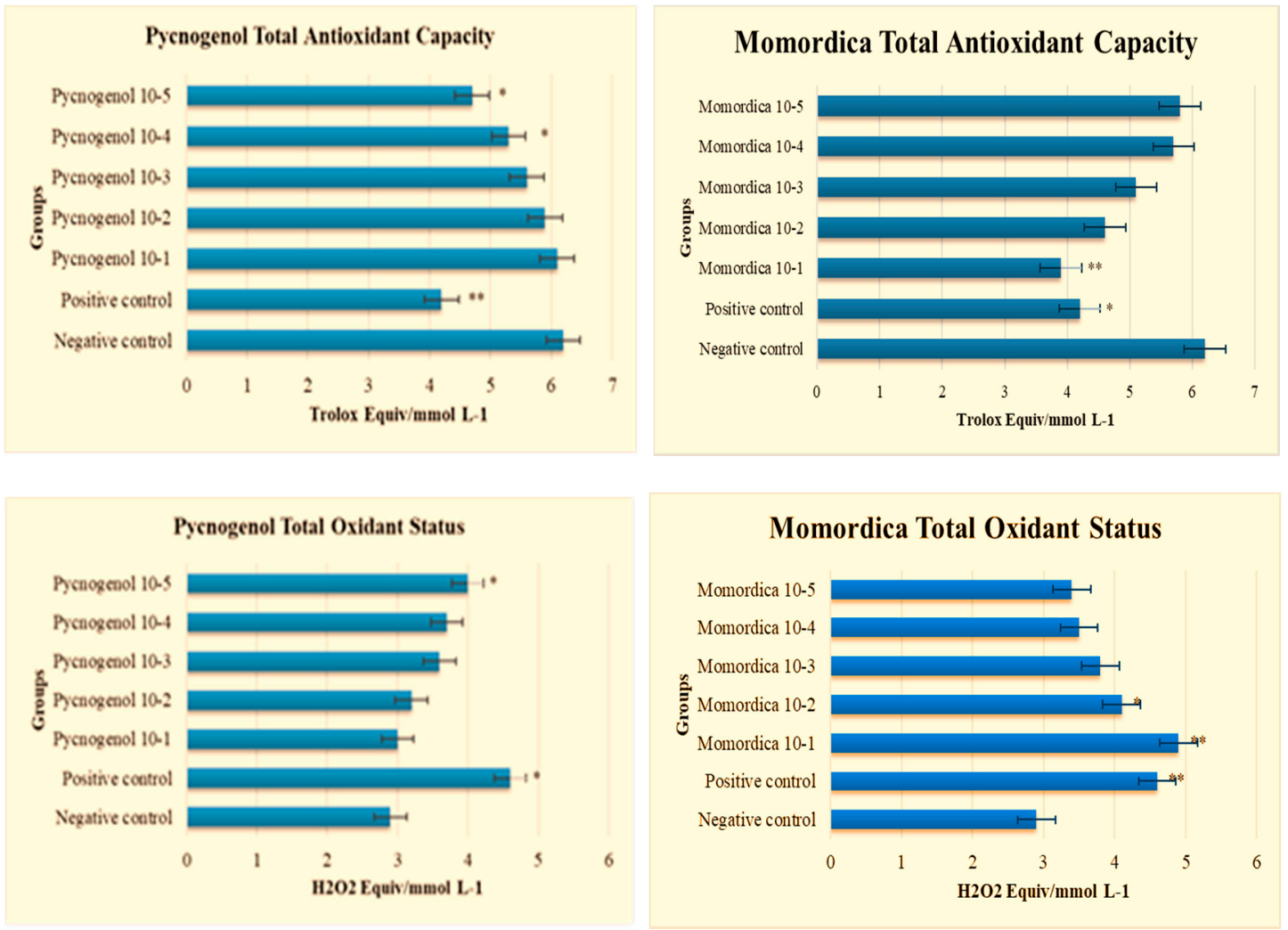

Figure 2. TAC and TOS of pycnogenol and momordica protective effect on induced imazamox toxicity effect on fibroblast cells ( $n=6$ /group) were shown. ${ }^{*}$ Significant differences at $p<0.05$ compared to control group; ${ }^{* *}$ Significant differences at the $p<0.001$ compared to control group.

\section{Discussion}

In conclusion momordica and pycnogenol effectively can be used in greenhouse worker toxicity. There is need to future study in laboratory animals to study chronic toxicity exposure of imazamox. In the present study, moderate necrotic and degenerative changes were detected in $250 \mu \mathrm{M}$ imazamox reported that both technical grade imazamox [7] as well as an $11.83 \%$ formulation of this herbicide caused a slight erythema on the skin [8]. This data has correlation with our data fibroblast degeneration was seen in high dose of imazamox herbicide. In addition, our result show momordica and pycnogenol in possible toxicity can effectively protects skin cels and reduced oxidation status.

Author Contributions: A.T. designed the experiments; A.T. and B.C. performed the experiments; A.T. analyzed the data; A.T. and B.C. wrote the paper.

Acknowledgments: All sources of funding of the study were met by the authors.

Conflicts of Interest: The authors declare no conflict of interest.

\section{References}

1. Dabrowski, J.M.; Shadung, J.M.; Wepener, V. Prioritizing agricultural pesticides used in South Africa based on their environmental mobility and potential human health effects. Environ Int. 2014, 62, 31-40.

2. He, Q.; Li, Y.; Li, H.; Zhang, P.; Zhang, A. Hypolipidemic and antioxidant potential of bitter gourd (Momordica charantia L.) leaf in mice fed on a high-fat diet. Pak. J. Pharm. Sci. 2018, 31, 118-128.

3. Packer, L.; Rimbach, G.; and Virgili, F. Antioxidant activity and biologic properties of a procyanidin-rich extract from pine (Pinus maritima) bark, pycnogenol. Free Radic. Biol. Med. 1999, 27, 704-724. 
4. Taghizadehghalehjoughi, A.; Hacimuftuoglu, A.; Cetin, M.; Ugur, A.B.; Galateanu, B.; Mezhuev, Y.; Okkay, U.; Taspinar, N.; Taspinar, M.; Uyanik, A.; et al. Effect of metformin/irinotecan-loaded poly-lactic-coglycolic acid nanoparticles on glioblastoma: In vitro and in vivo studies. Nanomedicine 2018, 13, 1595-1606.

5. Erel, O. A novel automated direct measurement method for total antioxidant capacity using a new generation, more stable ABTS radical cation. Clin Biochem. 2004, 37, 277-285.

6. Erel, O. A new automated colorimetric method for measuring total oxidant status. Clin Biochem. 2005, 38, 1103-1111.

7. Fischer, J. Skin Irritation Study in Albino Rabbits with AC 299,263 Technical: Lab Project Number: T-0519; New Jersey; American Cyanamid Co.: Wayne, NJ, USA, 1992.

8. Boczon, L. Skin Irritation Study in Albino Rabbits with AC 299,263 1AS Formulation: Lab Project Number: T0645: A 94-10; New Jersey; American Cyanamid Co.: Wayne, NJ, USA, 1994.

(C) 2018 by the authors. Licensee MDPI, Basel, Switzerland. This article is an open access article distributed under the terms and conditions of the Creative Commons Attribution (CC BY) license (http://creativecommons.org/licenses/by/4.0/). 